\title{
Aligned core-shell nanofibers delivering bioactive proteins
}

\section{IC Liao', SY Chaw² \& KW Leong ${ }^{2 \dagger}$}

${ }^{\dagger}$ Author for correspondence 1D epartment of Biomedical Engineering, D uke University, 136 H udson $\mathrm{H}$ all, Box 90281, D urham, NC 27708, USA

Tel.: +1 9196608466

Fax: +19196844488 kam.leong@duke.edu ${ }^{2} \mathrm{D}$ epartment of $\mathrm{M}$ aterials Science \& Engineering, Johns H opkins U niversity, Baltimore, M D 21218, USA

\begin{abstract}
Aims: Continuous nanostructures embedded with proteins may synergistically present topographical and biochemical signals to cells for tissue engineering applications. This study presents the co-axial electrospinning of aligned poly( $\varepsilon$-caprolactone) nanofibers encapsulated with bovine serum albumin and platelet-derived growth factor-bb for demonstration of controlled release and bioactivity retention, respectively.

Materials \& methods: Controllable release kinetics is achieved by incorporation of poly(ethylene glycol) as a porogen in the shell of the nanofibers. Results $\boldsymbol{\&}$ discussion: Poly(ethylene glycol) leaches out in a concentration- and molecular weight-dependent fashion, leading to bovine serum albumin release half-lives that range from 1 to 20 days. Optimized platelet-derived growth factor-bb-encapsulated nanofibers can completely release the protein with near zero-order kinetics and preserved bioactivity. Conclusion: Co-axial electrospinning is shown to be a versatile technique in achieving the delivery of biochemical signals in a controlled manner for regenerative medicine applications.
\end{abstract}

The complex process of tissue morphogenesis involves the coordinated delivery of biochemical and topographical cues. N anofibrous meshes can provide nanotopographical cues that stimulate cells in a manner drastically different from that of films and microscale fibrous scaffolds [1-6]. For instance, scanning electron microscope (SEM) images of hepatocytes cultured on electrospun nanofibers show a significantly greater degree of cell-substrate integration than cells seeded onto films [1]. To further augment the biofunctionality of nanofibers for tissue engineering scaffold design, co-axial electrospinning has been proposed to incorporate drug-delivery function into the nanofibrous meshes [6-9]. However, the degree of control over the release kinetics from these co-axially electrospun fibers has been limited. In this study, we propose the incorporation of a porogen into the shell of nanofibers to gain fine control over the rate of drug release.

We have opted to focus on poly(ethylene glycol) (PEG) as the porogen since it is noncytotoxic and easily filtered by the kidney at $\mathrm{M} \mathrm{w}$ of less than 10,000. It has also been applied as a porogen in a poly( $\varepsilon$-caprolactone) (PCL) film [10]. The wide range of PEG molecular weights available affords an opportunity to finely manipulate the nanoporous structure of the nanofiber shell. In this study, the characteristics of these biofunctional nanofibers are presented with respect to protein release kinetics, bioactivity of the released proteins and fiber alignment.
Materials \& methods

Co-axially electrospun PCL fibers were generated using a syringeinsidesyringe design (Figre1). Bovine serum albumin (BSA) was used as the model protein for initial optimization. BSA solution ( $M$ w 66318; Sigma, USA) at $10 \mathrm{mg} / \mathrm{ml}$ in distilled water was used as the core solution and 10\% w/v PCL (M n 42,500; Sigma, USA) in 60:40 (v/v) dichloromethane:ethanol was used as the shell solution. The needle gauges (G) used for dispensing the polymer shell and protein core solutions were 20 and $30 \mathrm{G}$, respectively. The flow rate was set at $1 \mathrm{ml} / \mathrm{h}$ for the core solution and $3 \mathrm{ml} / \mathrm{h}$ for the shell solution. The optimum flow rate ratio between the shell and core solutions was 3:1. Any large deviation from this ratio resulted in either a low protein encapsulation efficiency or electrospraying. The voltage gradient was adjusted from 10 to $15 \mathrm{kV}$ until the formation of a Taylor cone, with the electrospinning distance fixed at $5 \mathrm{~cm}$. Alignment of the core-shell PCL fibers was achieved by using a rotating drum ( 2000 RPM ) as the grounded target. D ifferent formulations of $P C L$ and PEG (M w of 1050, 3400 or 8000 and concentrations of 1 or $20 \mathrm{mg} / \mathrm{ml}$; Union Carbide Corporation, USA) were studied. Protein encapsulation was achieved by simply using a protein solution as the core solution. The electrospinning process was continued until $100 \mu \mathrm{l}$ of BSA (10 mg/ml) solution was encapsulated into the cores of the nanofibers, with the theoretical encapsulated amount being $1 \mathrm{mg}$ per sample. 


\section{Figure 1. Co-axial electrospinning setup.}

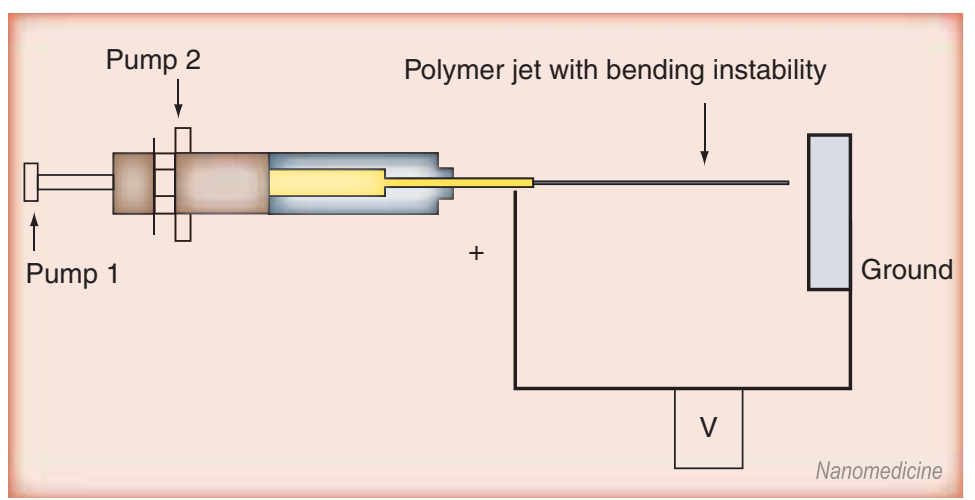

A syringe-inside-a-syringe is designed to allow two separate syringe pumps to control the flow of the core and shell solutions. The positive voltage from the voltage supply is connected to the needle tip, while the ground is connected to a rotating drum that collects the electrospun fibers.
The encapsulation of recombinant human platelet derived growth factor-bb (PDGF; Cordis Corporation, USA) was performed using the same technique. The electrospinning process was continued until $100 \mu \mathrm{l}$ of PDGF-bb $(200 \mu \mathrm{g} / \mathrm{ml})$ was dispensed to load each scaffold with $20 \mu \mathrm{g}$ of the growth factor. The average weight of the electrospun scaffold after drug loading was $6 \mathrm{mg}$. The encapsulation efficiency is defined as the percentage of the amount of protein detected over the theoretical amount of protein that should be encapsulated in the fibrous sample. In this work, we have focused on the release of a minute amount of protein (<1 mg) per sample, since most therapeutic proteins would be applied at the microgram level for tissue engineering.

For the protein release kinetics study, fibrous scaffolds $(n=3)$ were each incubated in $2 \mathrm{ml}$ of phosphate buffered solution (PBS; pH 7.4) at $37^{\circ} \mathrm{C}$, without shaking or stirring. The supernatant was then removed and replenished with fresh PBS solution at predetermined time intervals. The amount of BSA present in the supernatant was determined by micro-BCA ${ }^{\mathrm{TM}}$ protein assay, while the amount of PD GF-bb released was analyzed by enzyme-linked immunosorbent assay (ELISA). ELISA assay of the concentration of PD GF-bb was performed according to the protocol provided by the company ( $R \& D$ systems, USA).

To assess the release rate of the porogen from the shell of the PCL nanofibers, $20 \mathrm{mg}$ of fluorescein isothiocyanate polyethylene glycol (FITC-PEG; M w 3400, N ektar, 93\% degree of substitution) was blended into the $10 \% \mathrm{w} / \mathrm{V} \mathrm{PCL}$ in 60:40 (v/v) dichloromethane: ethanol. The electrospun fibrous scaffold made from this formulation was first dried under vacuum for 2 days before being incubated in $2 \mathrm{ml}$ of PBS solution at $37^{\circ} \mathrm{C}$. The supernatant was removed and replenished with fresh PBS solution at predetermined time intervals. The amount of FITC-PEG present in the supernatant was determined using ultraviolet (UV) spectrometry (Bio-rad) at an absorbance wavelength of $495 \mathrm{~nm}$ with a correction wavelength of $515 \mathrm{~nm}$.

The bioactivity of the released PDGF-bb was measured by the proliferation of N IH $3 \mathrm{~T} 3$ fibroblasts. The concentration of PDGF-bb released from the $P C L$ fibers $(n=3)$ was first determined using ELISA. The proliferation of 3T 3 fibroblasts incubated with the addition of $10 \mathrm{ng}$ of PDGF-bb released from the fibers was compared with those cultured in medium containing $10 \mathrm{ng} / \mathrm{ml}$ of fresh PD G F-bb (positive control), as well as those in medium only (negative control).

The FIT C-BSA-loaded PCL fibers were used to study fiber alignment and the quality of protein encapsulation. The size and surface morphology of the PCL fibers were analyzed using SEM and the core-shell structure was verified by transmission electron microscopy (TEM).

\section{Results}

In this study, the characteristics of co-axial nanofibers were studied with respect to protein distribution, protein release kinetics with respect to presence of porogen, bioactivity of the released protein and fiber alignment. Since the inner solution (BSA in water) is not electrospinnable and has a tendency to spray into droplets, the idea behind this work is to use the outer shell polymer to encapsulate the proteins. Therefore, the feed rate ratio is an important factor deciding the protein encapsulation efficiency and the protein-loading level of the resulting fibrous scaffold. Below a feed ratio of 3:1 outside/inside solution, loading efficiency is lowered owing to loss of the inner solution to electrospraying. H owever, loading level is also directly proportional to the feed rate ratio of the polymer solution, where approximately $100 \%$ loading efficiency is achieved with a 3:1 outside/inside solution feed ratio.

Figure $\mathbf{2 B}$ reveals the presence of the core-shell feature in the nanofibers through the encapsulation of $1 \% \mathrm{w} / \mathrm{v}$ uranyl acetate. The average diameter of the nanofibers is approximately $500 \mathrm{~nm}$, with an average core diameter of $250 \mathrm{~nm}$. The 
Figure 2. Transmission electron microscopy image of co-axial electrospun nanofibers.

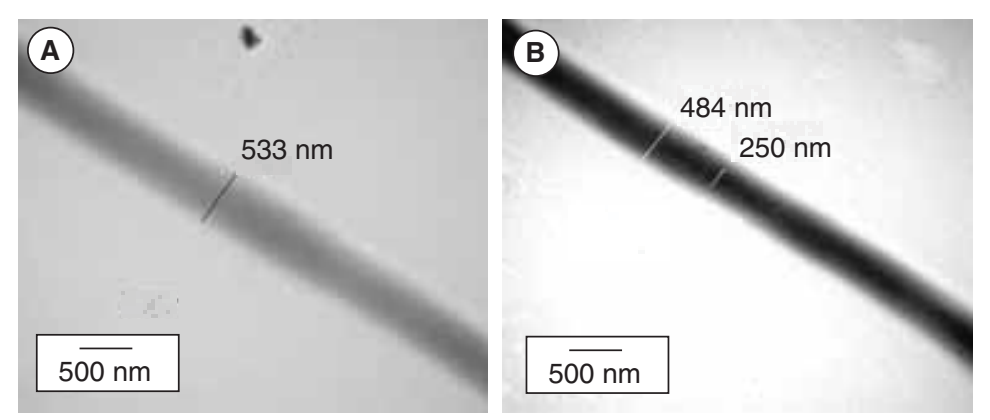

(A \& B) Transmission electron microscopy images illustrating the presence of core-shell feature in (A) fibers without uranyl acetate and (B) fibers with uranyl acetate.
The controlled release of a model growth factor was demonstrated by encapsulating PDGF-bb into PCL and PCL/PEG $(20 \mathrm{mg} / \mathrm{ml}$ of $M$ w 3400 PEG) nanofibers with $40 \%$ efficiency (Figre 5B). The encapsulated growth factor reached $100 \%$ release in 35 days with a relatively linear release profile (Figure5A). By contrast, only a very small amount of growth factor $(<1 \%)$ was released from PCL core-shell nanofibers without PEG in the shell.

\section{Discussion}

In this work, the control of drug release is designed around PEG's function as a porogen in the shell of the protein-loaded nanofibers. Low molecular weight PEG shave been shown to be noncytotoxic, filterable by kidneys and able to function as a porogen, creating pores in the scale of $500 \mathrm{~nm}$ [11-13]. By incorporating PEG into the shell of $P C L$ nanofibers to induce pore formation and possibly fiber swelling, we hypothesize that it can control the release of the encapsulated proteinsin a manner that is independent of loading concentration and core diameter. TEM images suggest the presence of the core-shell feature in the nanofibers (Figre 2B) with an average core diameter of $250 \mathrm{~nm}$. Previous literature suggest that the core-shell diameter ratio can be a function of the flow rate ratio between the two solutions, however, this phenomenon is not investigated in this work [7-9].

The changes in surface morphology and fiber diameter of nanofibers produced with different PCL/PEG formulations (M w 1050, 3400 or 8000) demonstrate that the incorporation of PEG increased fiber swelling and produced noticeable pore formation. The SEM images reveal that the most noticeable swelling and pore formation took place in the PCL/PEG 3400 composition, while less swelling and pore formation took place with the PCL/PEG 1050 and 8000 blends (Figre3). It can be suggested that PEG 1050 has an insignificant influence on the surface morphology as it is leached out of the nanofibers. H owever, the lack of swelling and pore formation with PCL/PEG 8000 may be attributed to the formation of a true PCL and PEG blend, decreasing the effectiveness of the PEG as a porogen (seen in PCL/PEG 3400). At day 30, the extent of swelling in PCL/PEG 3400 fibers decreased significantly compared with that observed in day 7. This sharp reduction in fiber diameter appears to be associated with the exhaustion of encapsulated drug in the core [7]. The result demonstrates that the effectiveness of the porogen is dependent on the concentration $(20>1 \mathrm{mg})$ and molecular 


\section{Figure 3. Scanning electron microscopy images of electrospun fibers incubated in phosphate buffered solution over a period of $\mathbf{2}$ weeks.}
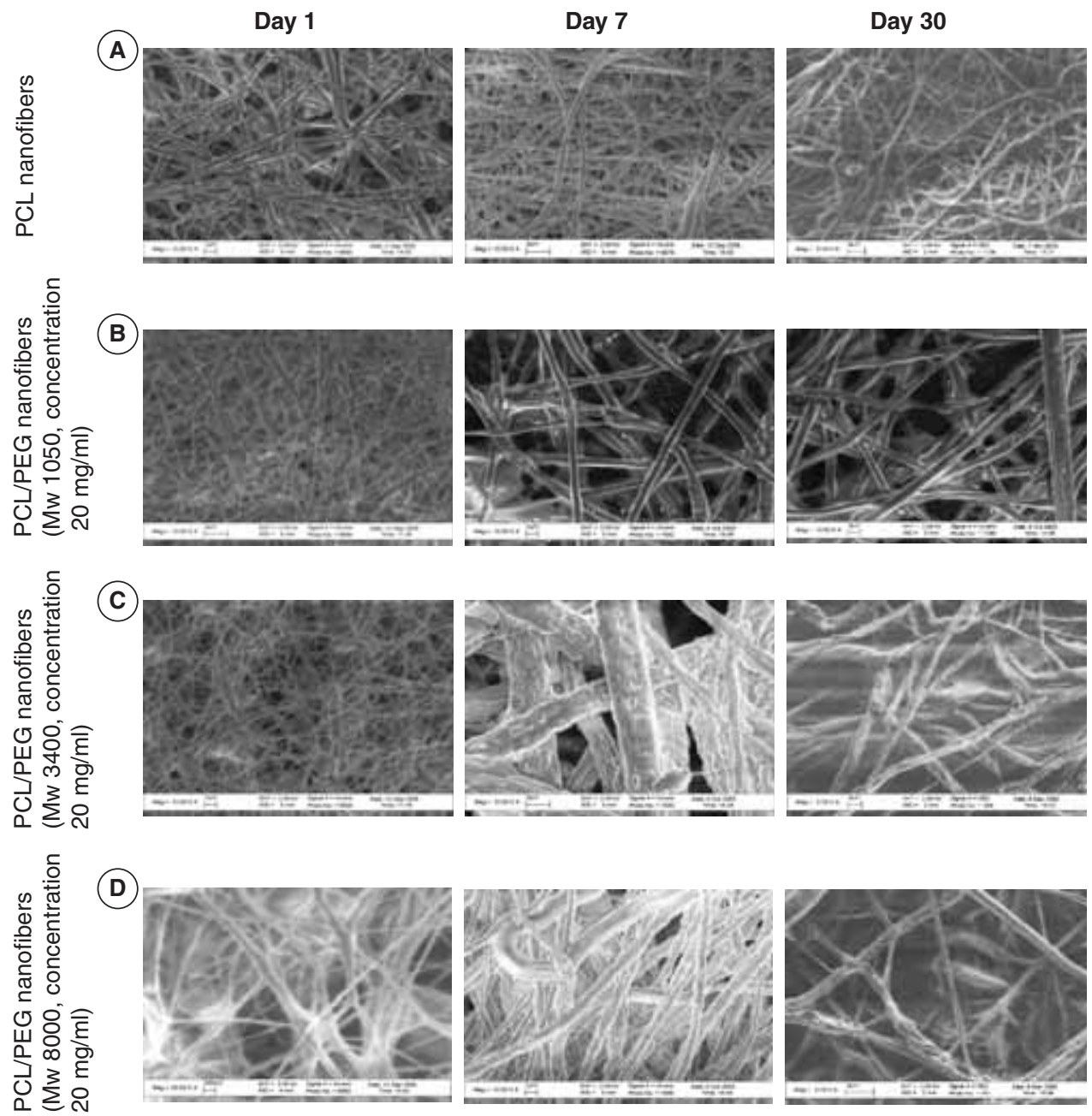

Scanning electron microscopy images of electrospun fibers with different PCL/PEG formulations. (A) Fibers (10\% PCL) at day 1, 7 and 30. (B) Fibers (10\% PCL $+20 \mathrm{mg} / \mathrm{ml} \mathrm{PEG} \mathrm{[M} \mathrm{w} \mathrm{1,000])} \mathrm{at} \mathrm{day} 1,7$ and 30 .

(C) Fibers (10\% PCL $+20 \mathrm{mg} / \mathrm{ml} \mathrm{PEG} \mathrm{[M} \mathrm{w} \mathrm{3400])} \mathrm{at} \mathrm{day} \mathrm{1,} 7$ and 30. (D) Fibers (10\% PCL $+20 \mathrm{mg} / \mathrm{ml}$ PEG [M w 8000]) at day 1,7 and day 30 .

PCL: Poly(e-caprolactone); PEG: Poly(ethylene glycol).

weight (3400 > $8000 \sim 1050)$. We speculate that the leaching of PEG 1000 is ineffective in forming pores, while PEG 8000 and PCL achieve a true polymer blend, which also decreases the ability of the porogen to form pores along the fibers.

The significance of controlling the rate of pore formation is demonstrated by the study of BSA release from different compositions of PCL/PEG fibers. BSA, often used as a filler protein or as a porogen in a drug-delivery vehicle, also served to induce its release from the core of the nanofibers ( $50 \%$ on day 40 ). Thisfinding agrees with previous publications on drug-encapsulated nanofibers [9]. The incorporation of PEG into PCL nanofibers increased the BSA rel ease rate, in a concentrationand molecular weight-dependent fashion (Figre4A). The FITC-PEG release profile (burst of $85 \%$ at day 1 ), SEM images of pore formation on the surface of the nanofibers and the increased burst of BSA release $(65 \%)$ at day 1 , indicating that the drug release takes place in a pore-dependent fashion. Theincorporation of porogen, as suggested by the changes in the release profiles of BSA, significantly improves the control over the protein release kinetics.

The quality of the controlled release nanofibers was examined through the encapsulation of FITC BSA. The fluorescent images reveal a uniform 
Figure 4. Encapsulation of BSA and FITC-BSA into poly( $\varepsilon$-caprolactone) nanoffibers.
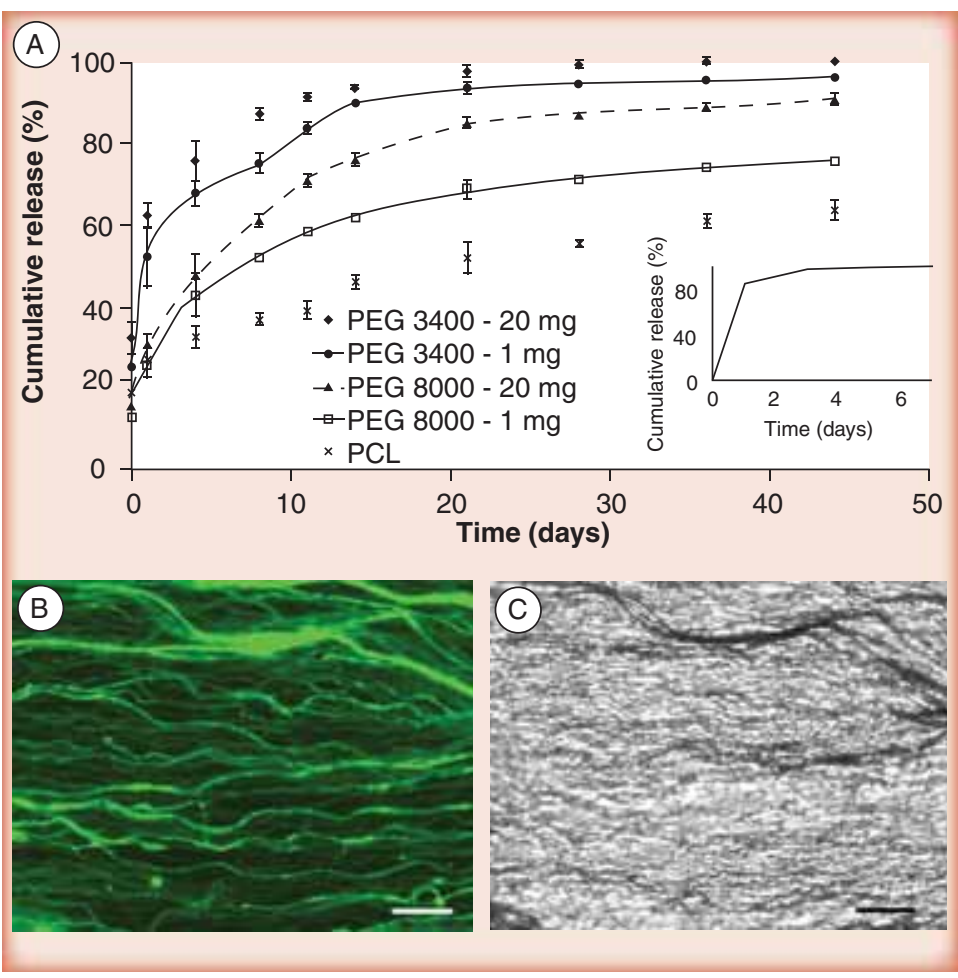

(A) Controlled release of encapsulated bovine serum albumin from PCL and various formulations of PEG-blended PCL nanofibers. PEG $3400-20 \mathrm{mg}: 10 \%$ PCL $+20 \mathrm{mg} / \mathrm{ml}$ of PEG (M w 3400). PEG $3400-1 \mathrm{mg}: 10 \%$ PCL $+1 \mathrm{mg} / \mathrm{ml}$ of PEG (M w 3400). PEG 8000 - $20 \mathrm{mg}: 10 \%$ PCL + $20 \mathrm{mg} / \mathrm{ml}$ of PEG (M w 8000). PEG $8000-1 \mathrm{mg}: 10 \% \mathrm{PCL}+1 \mathrm{mg} / \mathrm{ml}$ of PEG (M w 8000). PCL: $10 \% \mathrm{PCL}$ in 60:40 DCM :ethanol. Insert: Release rate of $20 \mathrm{mg}$ of FITC-PEG (M w 3400) from the shell of PCL/FITC-PEG nanofibers. (B) Fluorescent microscopy image of the aligned FITC-BSA-loaded PCL fibers. (C) Corresponding phase image of the aligned FITC-BSA-loaded PCL fibers. The scale bars are $20 \mu \mathrm{m}$.

BSA: Bovine serum albumin; FITC: Fluorescein isothiocyanate;

PCL: Poly( $\varepsilon$-caprolactone); PEG: Poly(ethylene glycol).

distribution of $B S A$, with no sign of aggregation and discontinuity of FITC-BSA within the core of the fibers (Figre $\mathbf{4 B} \boldsymbol{\&} \mathbf{C}$ ). The encapsulation efficiency $(\sim 100 \%)$ and the loading level $(5 \%$ of the fiber weight) in thisstudy demonstrate that co-axial electrospinning can significantly improve the protein-loading ability of electrospun fibers [14]. Fluorescent images also demonstrate that fiber alignment is possible. Aligned nanofibers have been shown to induce cell alignment on the surface of the nanofibers and even enhance the extracellular matrix production in fibroblasts [15]. Achieving alignment in protein-loaded nanofibers can therefore influence cellular orientation through nanotopographical cues and enhance cell differentiation and matrix production through biochemical cues delivered by the encapsulated growth factor.
Another significant finding in this work is the preservation of the bioactivity of the encapsulated growth factor. PD G F-bb was encapsulated into PCL and PCL/PEG $(20 \mathrm{mg} / \mathrm{ml}$ of $M$ w 3400 PEG) nanofibers, with very different release kinetics, with/without the presence of porogen. This can be attributed to the amount of protein distributed in the shell of the nanofibers, which can subsequently leave pores and channels in the shell after release. Unlike BSA, which can be loaded at a high level, the low amount of PDGF-bb in the core, owing to cost and potency, limits its partitioning to the shell during electrospinning. The release of such expensive and potent growth factors must there fore rely on pore formation on the fiber surface. The bioassay based on the proliferation rate of $\mathrm{NIH}$ 3T 3 cells also revealed the preservation of PD GF-bb bioactivity (Figure5B).

In this work, co-axial electrospinning is shown to be a process that can efficiently produce aligned nanofibers with encapsulated proteins. The controlled release of protein from the core of co-axial nanofibers first involves the leaching of PEG from the shell of the fibers, which then induces the solubilization and release of the encapsulated protein. M olecular weight and concentration of the incorporated porogen play major roles in the rate of protein release. The inclusion of a porogen (PEG) in the shell of the nanofibers provides versatility and control in the release of the drug of interest. Zhang and colleagues found that albumin can be released from the core of electrospun nanofibers and suggested that albumin served as its own porogen by partitioning a small amount of the protein in the organic phase of the fiber [9]. However, this approach would not work when dealing with expensive growth factors, as demonstrated in the release of PD GF-bb in this study (Figure5). The inclusion of PEG as a porogen allows the release of the protein of interest independent of the core diameter or protein type.

The co-axial electrospinning technique can also be applied to the encapsulation of particles or viruses, where the release will be dependent on the pore formation on the surface of nanofibers. Future advancement in this area could involve studying the cell-substrate behavior in the presence of porogen. In this work, PEG is not grafted onto the PCL polymer but is instead mixed into the PCL solution, eliminating brush effect formed by the PEG to inhibit cell attachment. H owever, cells seeded on the scaffold may possibly experience dynamic surface properties. It is also interesting to apply the 


\section{Figure 5. Controlled release and bioactivity of encapsulated} PDGF-bb $(20 \mu \mathrm{g})$ from electrospun nanofibers.

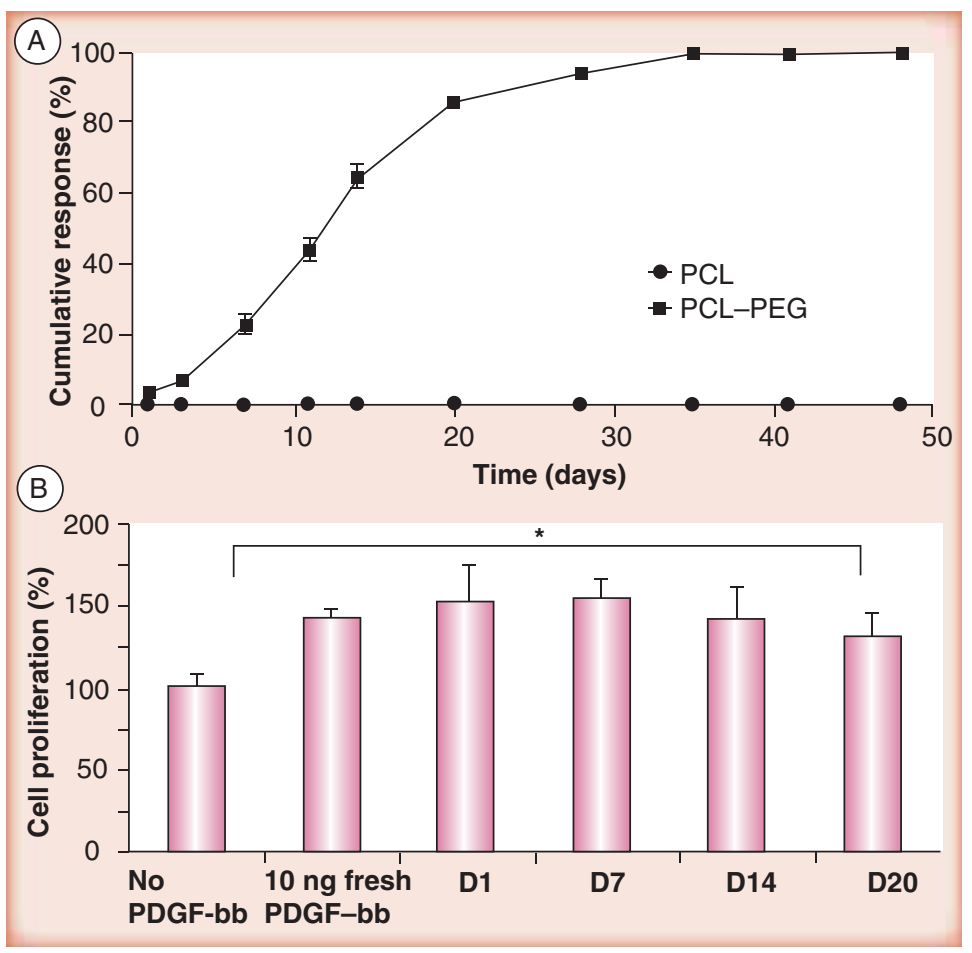

(A) Controlled release of encapsulated PDGF-bb from PCL and PCL-PEG (10\% $P C L+20 \mathrm{mg} / \mathrm{ml}$ PEG [M w 3400]). (B) Bioactivity of PDGF-bb released into the supernatant solution as determined by the enhanced proliferation rate of NIH 3T3 cells. Cell proliferation is normalized to negative control (no PDGF-bb). D1, 7, 14 and D20 refers to the supernatant solutions collected at day 1, 7, 14 and 20.

$* p<0.05$.

PCL: Poly(ع-caprolactone); PDGF: Platelet-derived growth factor; PEG: Poly(ethylene glycol).

\section{Conclusion}

Co-axial electrospinning was shown in this work to be an efficient method of encapsulating protein into nanofibers without compromising its bioactivity. By introducing PEG into the shell of $P C L$ fibers to serve as a porogen, the rate of protein release could be controlled by the molecular weight and concentration of PEG. The proteinloaded fibers could also be aligned to provide nanotopographical cues to the seeded cells. Such aligned, protein-loaded nanofibers may serve as basic units for the construction of anisotropic and biofunctional scaffolds to facilitate engineering of tissues with directionality.

\section{Future perspective}

Co-axial electrospinning is a versatile technique to prepare submicron core-shell fibers. Encapsulation of active agents in the core of these fibers, provided there is communication between the inside and outside of the fibers, can impart interesting functionalities to these structures. In regenerative medicine applications, delivery of biochemical signals to the cultured cells in a local and controlled manner may be highly beneficial. W ith the inherent advantage of high surface-to-volume ratio, the proposed fibers endowed with nanoscale features and bioactivity represent an attractive structural unit to build a scaffold that can mimic the extracellular matrix. N evertheless, there remains ample room to improve on the proposed technology. A deeper understanding, experimentally and theoretically, on how the different electrospinning parameters can influence the characteristics of the resulting core shell fibers will behighly valuable. What is the limit in size of these submicron scaled core-shell fibers? H ow can we collect these core-shell fibers into 3D structures with controlled architecture? H ow applicable is this technique to different polymers and bioactive agents? We expect co-axial electrospinning to be pursued increasingly by biomedical researchers for years to come.

\section{Executive summary}

- Electrospun nanofibers may enhance cell-substrate interactions, facilitating the development towards a nanostructured biomimetic tissue. Co-axial electrospinning enables the encapsulation of protein within the electrospun nanofibers, resulting in a scaffold that can offer biochemical and nanotopographic cues.

- In this study, we demonstrated that the protein of interest can be distributed uniformly throughout the fibers with a high loading level and that the co-axially electrospun fibers can be aligned to offer nanotopographic cues.

- Incorporation of a hydrophilic poly(ethylene glycol) (PEG) porogen within the shell phase of the core-shell nanofibers renders the fibers permeable to proteins by inducing swelling and pore formation. The protein release kinetics can be finely controlled by varying the nature and amount of the PEG in the shell of the nanofibers.

- The controlled release of platelet-derived growth factor-bb encapsulated in a small quantity $(1 \mu \mathrm{g})$ is dependent on the presence of porogen in the nanofibers. The released growth factor maintains its bioactivity as validated by a biological assay. 


\section{Aligned core-shell nanofibers delivering bioactive proteins - RESEARCH ARTICLE}

\section{Bibliography}

1. Chua KN , Lim W S, Zhang P et al.: Stable immobilization of rat hepatocyte spheroids on galactosylated nanofiber scaffold. Biomaterials 26, 2537-2547 (2005).

2. Yim EK, Reano RM, Pang SW, Yee AF, Chen CS, Leong KW: N anopattern-induced changes in morphology and motility of smooth muscle cells. Biomaterials 26, 5405-5413 (2005).

3. Rho KS, Jeong L, Lee G : Electrospinning of collagen nanofibers: effects on the behavior of normal human keratinocytes and earlystage wound healing. Biomaterials 8 , 1452-1461 (2006).

4. M atthews JA, W nek GE, Simpson DG, Bowlin GL: Electrospinning of collagen nanofibers. Biomacromolecules 3, 232-238 (2002).

5. Lim JY, $H$ ansen JC, Siedlecki CA: $O$ steoblast adhesion on poly(L-lactic acid)/polystyrene demixed thin film blends: effect of nanotopography, surface chemistry, and wettability. Biomacromolecules 6 , 3319-3327 (2005).
6. Ito $\mathrm{Y}, \mathrm{H}$ asuda $\mathrm{H}, \mathrm{K}$ amitakahara $\mathrm{M}: \mathrm{A}$ composite of hydroxyapatite with electrospun biodegradable nanofibers as a tissue engineering material. J. Biosci. Bioeng. 100, 43-49 (2005).

7. Jiang $\mathrm{H}, \mathrm{Hu} Y, \mathrm{Li} Y$, Zhao P, Zhu K, Chen $\mathrm{W}$ : A facile technique to prepare biodegradable coaxial electrospun nanofibers for controlled release of bioactive agents. J. Control. Release 108, 237-243 (2005).

8. Huang ZM , H eCL, Yang A: Encapsulating drugs in biodegradable ultrafine fibers through co-axial electrospinning. J. Biomed. $M$ ater. Res A (2006) (In Press).

9. Zhang $Y Z$, Wang $X$, Feng $Y, L i$ J, Lim CT, Ramakrishna S: C oaxial electrospinning of (fluorescein isothiocyanate-conjugated bovine serum albumin)-encapsulated poly ( $\varepsilon$-caprolactone) nanofibers for sustained release. Biomacromolecules 7 , 1049-1057 (2006).

10. Luong-Van E, Grondahl L, Chua KN , Leong KW, N urcombe V, Cool SM : Controlled release of heparin from poly( $\varepsilon$-caprolactone) electrospun fibers. Biomaterials 27, 2042-2050 (2006).
11. Lu CH , Lin W J: Permeation of protein from porous poly(epsilon-caprolactone) films. J. Biomed. M ater. Res. 63, 220-225 (2002)

12. H edberg EL, Tang A, Crowther RS, Carney D H, M ikos AG : Controlled release of an osteogenic peptide from injectable biodegradable polymeric composites. J. Control. Release 84, 137-150 (2002).

13. Kim H K, Chung HJ, Park TG : Biodegradable polymeric microspheres with "open/closed" pores for sustained release of human growth hormone. J. Control. Release 112, 167-174 (2006).

14. Chew SY, Wen J, Yim EK, Leong KW: Sustained release of proteins from electrospunbiodegradable fibers. Biomacromolecules 6, 2017-2024 (2005).

15. Lee CH, Shin HJ, Cho IH : N anofiber alignment and direction of mechanical strain affect the ECM production of human ACL fibroblast. Biomaterials26, 1261-1270 (2005). 